\title{
Surgical reconstruction of massive scrotal lymphedema associated with hidradenitis suppurativa: a case report
}

\author{
Ziyou Yü, Lingling Sheng", Weigang Cao, Shengli Li, Zhaohua Jiang \\ Department of Plastic and Reconstructive Surgery, Shanghai 9th People's Hospital, Shanghai Jiao Tong University, Shanghai, China \\ "These authors contributed equally to this work. \\ Correspondence to: Zhaohua Jiang. Shanghai Jiao Tong University, 639 Zhi Zao Ju Road, Shanghai 200011, China. Email: Dr_zhjiang@126.com.
}

\begin{abstract}
Hidradenitis suppurativa (HS) is a chronic, recurrent inflammatory cutaneous disease affecting apocrine glands. It can be associated with lymphedema of the surrounding tissues and most commonly affects scrotum. As a debilitating complication of HS, lymphedema can cause significant morbidity and further exacerbate HS condition, thus causing a vicious cycle. Surgery was reported to be the most common treatment for this complication. Here, we present a 41-year-old patient with massive scrotal lymphedema following a 2-year history of HS. To reduce the volume of the scrotal mass and improve the appearance and function of the scrotum, we modified the Charle's procedure by complete excision of the affected tissue while retaining the scrotal septum, preserving the subcutaneous lymphatic tissue flap, turnover of the perididymis, and primary closure. We found that this approach achieved satisfactory cosmetic results, maintained cutaneous sensation and restored erections. There was no adverse event following surgery. No recurrence occurred over 6-month of follow-up. We believe that this modified Charles' procedure can improve the morphology and lymphatic function of the scrotum and recommend its use whenever possible. While rare, HS associated lymphedema should alert clinician to the potential consequence of an advanced disease situation. Collaborative approach with surgery in the management of this condition should be considered at early stage.
\end{abstract}

Keywords: Scrotal lymphedema; hidradenitis suppurativa (HS); surgical reconstruction; case report

Submitted Apr 15, 2020. Accepted for publication Nov 21, 2020.

doi: $10.21037 /$ gs-20-460

View this article at: http://dx.doi.org/10.21037/gs-20-460

\section{Introduction}

Hidradenitis suppurativa (HS) is a chronic, recurrent inflammatory cutaneous disease affecting apocrine glands (1). Repeated episodes of HS can lead to abscesses, sinus tracts, scarring, contracture, obstruction of the lymphatics and impaired lymph flow which causes chronic lymphedema at late stage. As one of the challenging complications of longstanding HS, lymphedema can cause significant morbidity and further complicated HS treatment (2). Although it has been reported that the prevalence of HS reached to approximately $1 \%$ of the population, lymphedema following HS is rare and published cases are few (3). The diagnosis of HS complicated lymphedema was based on medical history and clinical features. It has been reported that though HS mostly affects axillae, lymphedema was not found in this region and most commonly occurs at scrotum $(4,5)$. Other implicated regions reported involve penis, labia majora, perineum, groin, etc. $(2,6,7)$.

Combined medical and surgical therapy were recommended for the treatment of HS. Conservative therapies such as topical and oral therapy have been used with favorable outcomes for mild to moderate HS. Immunotherapy is also helpful. For more advanced situation and HS complicated lymphedema, surgery was the most commonly reported treatment with good outcomes (8). Surgical excision of the thickened tissue with reconstruction has shown favorable results such as the return of skin sensation, voiding and erectile function, improved quality of life. Adverse events related to surgery includes mild penile edema and small wound opening. 

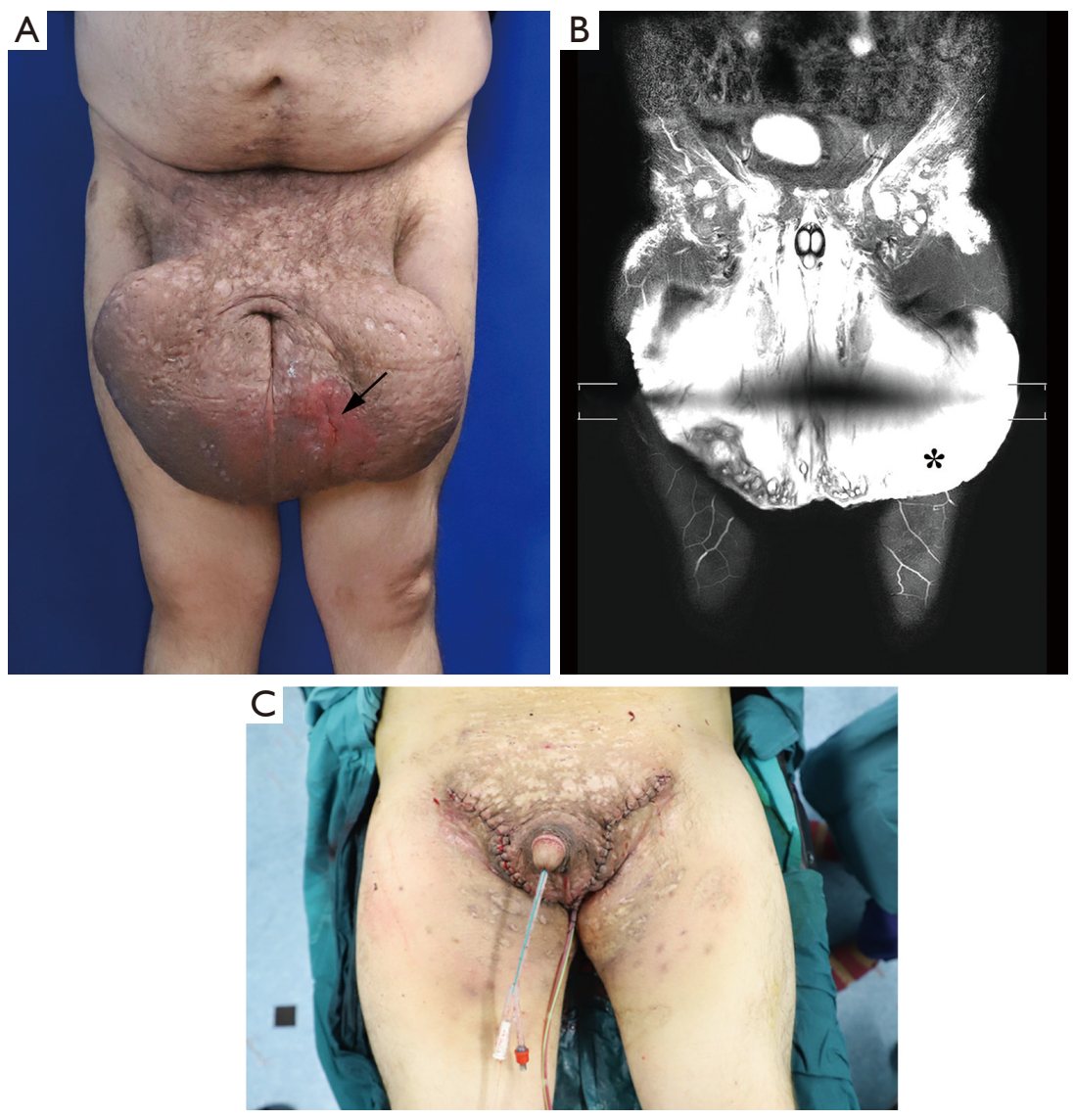

Figure 1 A 41-year-old man with 2-year progressive scrotal lymphedema associated with hidradenitis suppurativa. (A) Preoperative appearance of the penoscrotal region. A $2 \mathrm{~cm}$ draining sinus tract was presented at the left scrotal skin (arrow); (B) magnetic resonance lymphography (MRL) obtained before surgery showed massive dermal reflux of the scrotum (asterisk). (C) immediate postoperative scrotal appearance showed evident size reduction of the scrotum.

Here, we present a case of massive scrotal lymphedema associated with HS, in whom the scrotal edema was assessed by magnetic resonance lymphangiography (MRL). The patient was treated with surgical reduction procedures and reconstruction of penis and scrotum. The scrotal septum and a subcutaneous lymphatic tissue flap was retained, which we believe can improve the appearance and lymphatic drainage function. We present the following case in accordance with the CARE reporting checklist (available at http://dx.doi.org/10.21037/gs-20-460).

\section{Case presentation}

A 41-year-old man was referred with a 2-year history of hidradenitis suppurativa of the scrotum accompanied with significant scrotal swelling, recurrent erysipelas, pain and suppuration, which affects his ability to walk. He had been treated previously for oral antibiotics including of cefuroxime, diosmin and doxycycline hydrochloride, but without resolution. He had recurrent hidradenitis suppurativa of axillae since the age of sixteen and has been treated with topical medications.

On examination, there was massively enlarged scrotum, which measured approximately $90 \mathrm{~cm}$ in circumferences, obscuring the penis (Figure 1A). Multiple draining sinus tracts were present causing constant weeping of the malodorous gray or brown liquid, with one sinus tract reach to $2 \mathrm{~cm}$. The urethral opening is in the upper middle of the enlarged scrotum. Testes and cord structures were not palpable. The perineum and axillae area was grossly indurated and scarred.

MRL was performed to assess the extent of lymphedema, 


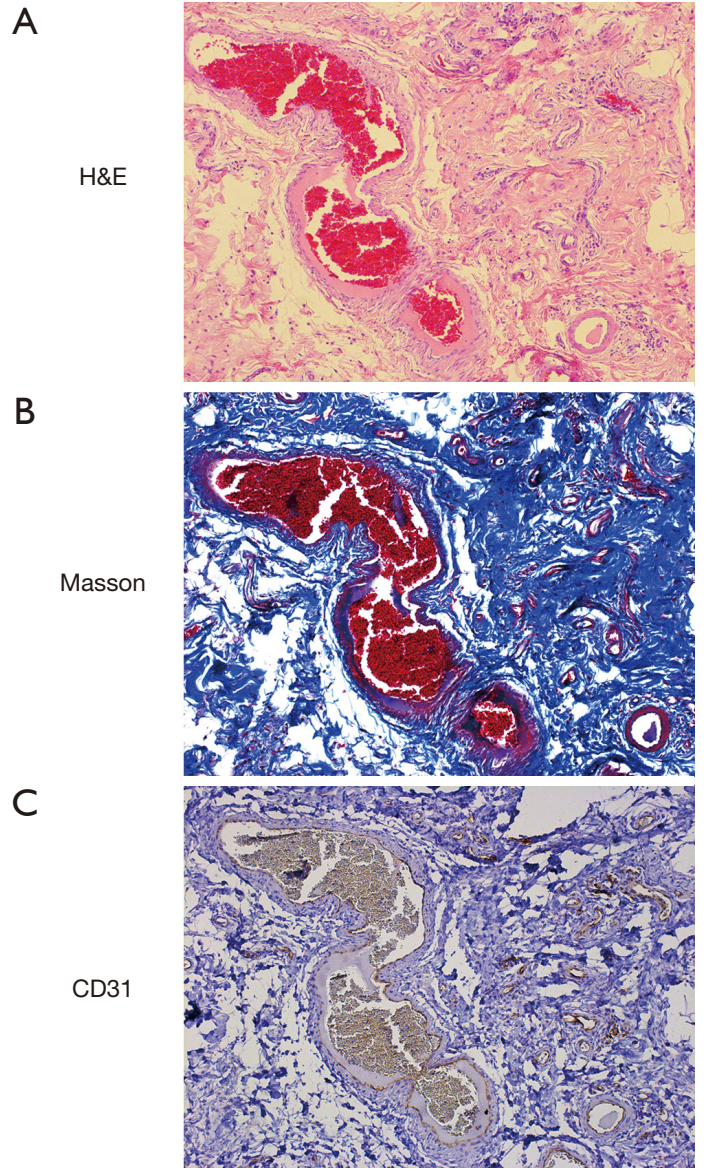

Figure 2 Pathological evaluation of the affected scrotal skin (20x). (A) $\mathrm{H} \& \mathrm{E}$ staining shows thickened dermis with inflammatory cell infiltration. (B) Masson staining reveal massive fibrosis of the edematous tissue. (C) Immunostaining of CD31 shows increased CD31+ blood vessel density.

as well as the morphology and function of the scrotum lymphatics. Thickened scrotal skin and subcutaneous tissue with massive edema were clearly visualized (Figure 1B). Blood chemistry revealed mild anemia with decreased hemoglobin $(99 \mathrm{~g} / \mathrm{L})$. Serologic levels of serum IgG $(22.8 \mathrm{~g} / \mathrm{L})$ and $\operatorname{IgE}$ $(256 \mathrm{IU} / \mathrm{mL})$ were increased at diagnosis.

The patient underwent radical excision of the scrotum after 1-week anti-infective treatment (ceftriaxone sodium $2 \mathrm{~g}$ and Metronidazole $500 \mathrm{mg}$ IV infusion per day). An incision was made to preserve the scrotal septum and subcutaneous tissue, maximizing the excision of the interstitial edema tissue. The inverted tunicae vaginalis was fixed with base of the scrotal tissue and separated by the scrotal septum. During surgery, a subcutaneous lymphatic tissue flap was retained and bridged to the affected side to maximize recovery and improve lymphatic drainage function. The remaining adjacent skin was sutured and fixed to the scrotal septum to cover the testis and seal the wound to reconstruct the scrotum. Satisfactory cosmetic results were achieved immediately after operation (Figure $1 C$ ).

Pathological examination of the resected specimen, which weighed $3.76 \mathrm{~kg}$, demonstrated multiple abscesses and sinus tracts in the subcutaneous tissue. Histological staining revealed focal infiltration of inflammatory cells and extensive fibrosis (Figure 2A,B). Immunostaining of CD31 revealed increased $\mathrm{CD} 31^{+}$capillaries (Figure $2 C$ ), whereas podoplanin (lymphatic endothelium specific marker) staining was absent (data not show).

The patient received methylprednisolone with $40 \mathrm{mg}$ IV infusion per day for 3 days after surgery. There was no adverse event following surgery. Over 6-month followup, the patient had no recurrence of lymphedema and skin inflammation (Figure 3A). At 6-month postoperatively, MRL revealed no signs of lymphedema (Figure 3B). Satisfactory cosmetic results, maintained cutaneous sensation as well as recovery of sexual function was achieved. A timeline of the patient's treatment course is shown in Figure 4.

All procedures performed in studies involving human participants were in accordance with the ethical standards of the institutional and/or national research committee(s) and with the Helsinki Declaration (as revised in 2013). Written informed consent was obtained from the patient.

\section{Discussion}

Lymphedema secondary to HS is rare and debilitating. Although it has been recognized that this complication is caused by chronic and recurrent inflammation due to HS and subsequent blockade of the lymphatic channels, the association between lymphedema and HS remains largely unclear (9-11). Lymphedema may lead to recurrent infections such as erysipelas and skin ulceration, which can further exacerbate HS condition and causing a vicious cycle. Although conservative therapy, such as complex decongestive therapy, is the most commonly applied approach in managing lymphedema, it was only reported for one patient with HS associated lymphedema and achieved improved outcomes (12). Medical therapy, which include minocycline, isotretinoin, adalimumab, etc. is important in managing the inflammatory conditions of HS and was recommended to apply in combination with surgery. Surgery was reported to be the most common treatment 

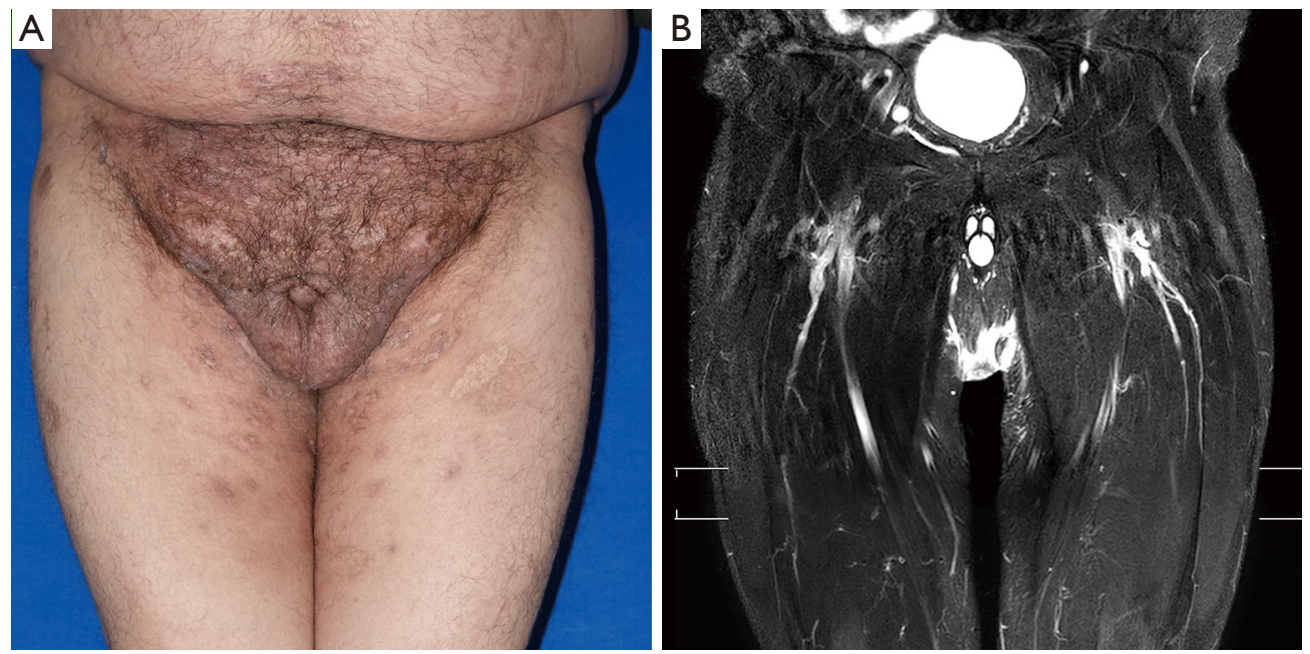

Figure 3 Postoperative appearance and MRL images of the scrotum at 6-month follow-up. (A) Clinical photograph of the scrotum 6-month after surgery. Note that no trend of recurrent lymphedema and HS of the scrotum. (B) MRL showed nearly normal scrotal skin and subcutaneous tissue.

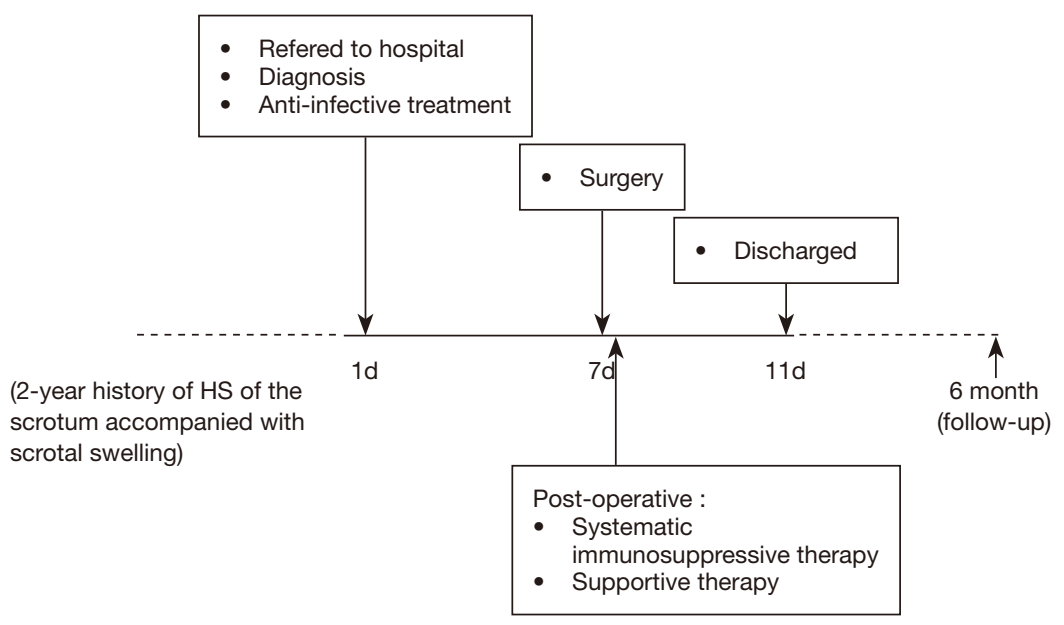

Figure 4 Patient's treatment timeline.

for this complication with favorable outcomes (8). Massive or radical excision of the diseased tissue covered with split thickness skin graft or skin flap was the most commonly performed surgical approach for treating lymphedema following HS $(5,13,14)$.

In this unusual case, we adopted modified Charles' procedure which we have introduced previously for primary genital lymphedema $(15,16)$. Improvements were made include discarding skin grafting and applied the adjacent scrotal and penile skin for suturing to protect superficial lymphatic tissue from damage, inversion of the tunica vaginalis to reduce the chance of testicular hydrocele, as well as retaining the scrotal septum and subcutaneous lymphatic tissue flaps to improve morphology and lymphatic function. We believe that these procedures are important to maintain the long-term efficacy and prevent the recurrence of lymphedema. The major limitation of our case report presentation is limited follow-up times.

While rare, HS associated lymphedema should alert clinician to the potential consequence of an advanced disease situation which may cause gross deformity. Collaborative approach with surgery in the management of 
this condition should be considered at early stage.

\section{Conclusions}

Lymphedema secondary to HS is a rare and debilitating complication due to the blockade of lymphatic channels. In this case report, we described a patient with massive scrotal lymphedema associated with chronic HS of the scrotum. Surgical excision and reconstruction was performed and achieved favorable functional and cosmetic results. We believe that retaining the scrotal septum and subcutaneous lymphatic tissue flaps can improve the morphology and lymphatic function of the scrotum and recommend its use whenever possible.

\section{Acknowledgments}

Funding: This work was supported by National Natural Science Foundation of China (81372080) and Natural Science Foundation of Shanghai (16ZR1419700).

\section{Footnote}

Reporting Checklist: The authors have completed the CARE reporting checklist. Available at http://dx.doi.org/10.21037/ gs-20-460

Conflicts of Interest: All authors have completed the ICMJE uniform disclosure form (available at http://dx.doi. org/10.21037/gs-20-460). The authors have no conflicts of interest to declare.

Ethical Statement: The authors are accountable for all aspects of the work in ensuring that questions related to the accuracy or integrity of any part of the work are appropriately investigated and resolved. All procedures performed in studies involving human participants were in accordance with the ethical standards of the institutional and/or national research committee(s) and with the Helsinki Declaration (as revised in 2013). Written informed consent was obtained from the patient.

Open Access Statement: This is an Open Access article distributed in accordance with the Creative Commons Attribution-NonCommercial-NoDerivs 4.0 International License (CC BY-NC-ND 4.0), which permits the noncommercial replication and distribution of the article with the strict proviso that no changes or edits are made and the original work is properly cited (including links to both the formal publication through the relevant DOI and the license). See: https://creativecommons.org/licenses/by-nc-nd/4.0/.

\section{References}

1. Alikhan A, Lynch PJ, Eisen DB. Hidradenitis Suppurativa: A Comprehensive Review. J Am Acad Dermatol 2009;60:539-61; quiz 562-3.

2. Chen ML, Odom B, Santucci RA. Surgical Management of Genitoperineal Hidradenitis Suppurativa in Men. Urology 2014;83:1412-7.

3. Jemec GB, Heidenheim M, Nielsen NH. The prevalence of hidradenitis suppurativa and its potential precursor lesions. J Am Acad Dermatol 1996;35:191-4.

4. Williams ST, Busby RC, DeMuth RJ, et al. Perineal hidradenitis suppurativa: presentation of two unusual complications and a review. Ann Plast Surg 1991;26:456-62.

5. Thornton JP, Abcarian H. Surgical treatment of perianal and perineal hidradenitis suppurativa. Dis Colon Rectum 1978;21:573-7.

6. Pacheco YD, García-Duque O, Fernández-Palacios J. Penile and Scrotal Lymphedema Associated With Hidradenitis Suppurativa: Case Report and Review of Surgical Options. Cir Cir 2018;86:84-8.

7. Musumeci ML, Scilletta A, Sorci F, et al. Genital Lymphedema Associated With Hidradenitis Suppurativa Unresponsive to Adalimumab Treatment. JAAD Case Rep 2019;5:326-8.

8. Micieli R, Alavi A. Lymphedema in Patients With Hidradenitis Suppurativa: A Systematic Review of Published Literature. Int J Dermatol 2018;57:1471-80.

9. Slade DE, Powell BW, Mortimer PS. Hidradenitis suppurativa: pathogenesis and management. Br J Plast Surg 2003;56:451-61.

10. Menter A. Recognizing and managing comorbidities and complications in hidradenitis suppurativa. Semin Cutan Med Surg 2014;33:S54-6.

11. Moosbrugger EA, Mutasim DF. Hidradenitis Suppurativa Complicated by Severe Lymphedema and Lymphangiectasias. J Am Acad Dermatol 2011;64:1223-4.

12. Moschella SL. Is there a role for infliximab in the current therapy of hidradenitis suppurativa? A report of three treated cases. Int J Dermatol 2007;46:1287-91.

13. Baughman SM, Cespedes RD. Unusual presentation of hidradenitis suppurativa with massive enlargement of penis. Urology 2004;64:377-8.

14. Konety BR, Cooper T, Flood HD, et al. Scrotal 
elephantiasis associated with hidradenitis suppurativa. Plast Reconstr Surg 1996;97:1243-5.

15. Lu Q, Jiang Z, Zhao Z, et al. Assessment of The Lymphatic System of the Genitalia Using Magnetic Resonance Lymphography Before and After Treatment

Cite this article as: Yu Z, Sheng L, Cao W, Li S, Jiang Z. Surgical reconstruction of massive scrotal lymphedema associated with hidradenitis suppurativa: a case report. Gland Surg 2021;10(5):1800-1805. doi: 10.21037/gs-20-460 of Male Genital Lymphedema. Medicine (Baltimore) 2016;95:e3755.

16. Yu Z, Sheng L, Cao W, et al. Surgical reconstruction of primary genital lymphedema-long term therapeutic efficacy. Gland Surg 2020;9:575-81. 